\title{
Association between weekly fruit and vegetable consumption and depressive symptoms: results from the Korean Elderly Environmental Panel study
}

\author{
Youjeong Yuk', Chae-Rin Han', Yoonyoung Jang ${ }^{2,3}$, Yun-Chul Hong ${ }^{2,3,4}$, Yoon-Jung Choi ${ }^{2,3}$ \\ ${ }^{1}$ Seoul National University College of Medicine, Seoul, Korea; '2Department of Preventive Medicine, Seoul National University College of \\ Medicine, Seoul, Korea; ${ }^{3}$ Environmental Health Center, Seoul National University College of Medicine, Seoul, Korea; ${ }^{4}$ Institute of Environmental \\ Medicine, Seoul National University Medical Research Center, Seoul, Korea
}

OBJECTIVES: Although previous studies have investigated the correlation between fruit and vegetable consumption and depression, the results remain inconclusive. The present study aimed to investigate the association between weekly fruit and vegetable consumption and depressive symptoms in elderly Koreans.

METHODS: A multiple covariate linear regression analysis was performed using the data of 1,226 elderly individuals $\geq 60$ years of age who participated in the Korean Elderly Environmental Panel II study from 2012 to 2014. Depressive symptoms were evaluated using the Korean version of the Short form Geriatric Depression Scale (SGDS-K). Generalized linear mixed-effects models were constructed to analyze the repeated measurements of 305 people who participated in the survey every year.

RESULTS: After adjusting for confounders, SGDS-K scores were negatively associated with the frequency of weekly fruit consumption as follows ( $\beta$ [ $95 \%$ confidence interval; $\mathrm{CI}$ ]: -0.17 [ -0.28 to -0.05 ], -0.17 [ -0.27 to -0.07 ], -0.42 [ -0.54 to 0.29 ], and -0.33 [-0.44 to -0.21]) for less than 1 time/wk, 1-3 times/wk, $4-6$ times/wk, and daily, respectively, compared to no consumption. The SGDS-K scores were also negatively associated with the frequency of vegetable consumption ( $\beta$ [ $95 \% \mathrm{CI}$ ]: -0.86 [-1.18 to -0.55$]$, -0.18 [ -0.35 to -0.01$],-0.36$ [ -0.53 to -0.18$]$, and -0.15 [ -0.29 to 0.00$]$ ) in the above order, respectively.

CONCLUSIONS: Fruit consumption was inversely associated with depression levels in a dose-dependent manner. Although there was no dose-response relationship between vegetable consumption and the level of depression, it was negatively associated with SGDS-K scores.

KEY WORDS: Depression, Fruit, Vegetables, Aged, Korean, Korean version of Short form Geriatric Depression Scale

\section{Correspondence: Yoon-Jung Choi}

Department of Preventive Medicine, Seoul National University College of Medicine, 103 Daehakro, Jongno-gu, Seoul 03080, Korea E-mail:hierica8@snu.ac.kr

Received: Feb 18, 2021 / Accepted: Apr 20, 2021 / Published: Apr 20, 2021

This article is available from: https://e-epih.org/

(c) This is an open-access article distributed under the terms of the Creative Commons Attribution License (https://creativecommons.org/licenses/by/4.0/), which permits unrestricted use, distribution, and reproduction in any medium, provided the original work is properly cited.

(C) 2021, Korean Society of Epidemiology

\section{INTRODUCTION}

Depression is a major public health problem with an increasing incidence and resultant global burden [1]. Other chronic diseases can exacerbate the symptoms of depression [2]. Hence, it is important to identify preventive or curative methods to prevent or relieve symptoms in patients with depression. Several previous studies have investigated the relationship between fruit and vegetable consumption and depressive symptoms. It is postulated that fruit and vegetable consumption may alleviate depressive symptoms, as they contain antioxidants and minerals that are involved in the synthesis of neurotransmitters. Nevertheless, the results are inconsistent [3-11], which could be attributed to variations in die- 
tary patterns among different countries. Consequently, each country should conduct individual studies to evaluate the effects of fruit and vegetable consumption on depression.

Korea has a high prevalence of depression and suicide. However, very few studies have investigated the association between fruit and vegetable consumption and depressive symptoms among elderly Koreans [12-15]. Among the Organization for Economic Cooperation and Development countries, Korea recorded the highest suicide rate among adults above the age of 70 years [16]. The incidence of depression increases exponentially with age [17]. In Korea, the highest suicide rate is observed among individuals above the age of 80 years, followed by individuals above the age of 70 years [16]. Elderly individuals have restrictions regarding physical and social activities and are at risk of consuming a nutritionally inadequate diet $[17,18]$. Hence, research should focus on the dietary behavior of the elderly, which can be easily improved. However, to the best of our knowledge, no previous study has focused solely on Korean elderly people regarding this particular aspect. The current study aimed to investigate the associations between weekly fruit and vegetable consumption and depressive symptoms in elderly Korean individuals.

\section{MATERIALS AND METHODS}

\section{Participants and data source}

The Korean Elderly Environmental Panel (KEEP) study is a community-based, repeated-measures survey that was established to study the effects of diverse environmental exposures on the health of elderly Koreans [19]. The study methods and details are described in the literature [19]. Briefly, the KEEP study is composed of the KEEP-I and the KEEP-II. In KEEP-I, 560 elderly subjects were re- cruited in Seoul and followed up five times (six surveys) between 2008 and 2010, and in KEEP-II, 800 elderly subjects were recruited (400 from Seoul and 400 from Asan) and followed up twice (three surveys). Our study used data from the KEEP-II, which was conducted annually from 2012 to 2014. Most of the questionnaires, including weekly fruit/vegetable intake and the Korean version of the Short form Geriatric Depression Scale (SGDS-K), were investigated every year. The study participants were elderly adults aged $\geq 60$ years with an appropriate cognitive level to understand the instructions of the survey, who regularly visited one of the two community welfare centers in the Seongbuk-gu area of Seoul and Asan, Korea. Of the 800 KEEP-II participants recruited in 2012, 416 and 327 participants were followed up in 2013 and 2014, respectively. New enrollment included 384 and 71 participants in 2013 and 2014, respectively. More details regarding follow-up status in Seoul and Asan are presented in Supplementary Material 1. From the baseline data of 1,255 individuals who participated in the study between 2012 and 2014, 5 participants whose response to the query regarding weekly fruit or vegetable consumption was "do not know," 1 participant without SGDS-K information, and 23 participants who were diagnosed with depression and undergoing treatment at the baseline were excluded. However, subjects with depression who were not receiving therapy were included. Among the study subjects, 305 individuals who participated in the KEEP-II for three consecutive years were selected for the repeated-measures analysis (Figure 1).

\section{Assessment of depression}

The current study used the SGDS-K as a screening tool to assess depression. The SGDS-K comprises 15 questions that evaluate the participant's mood in the preceding week. The participants choose

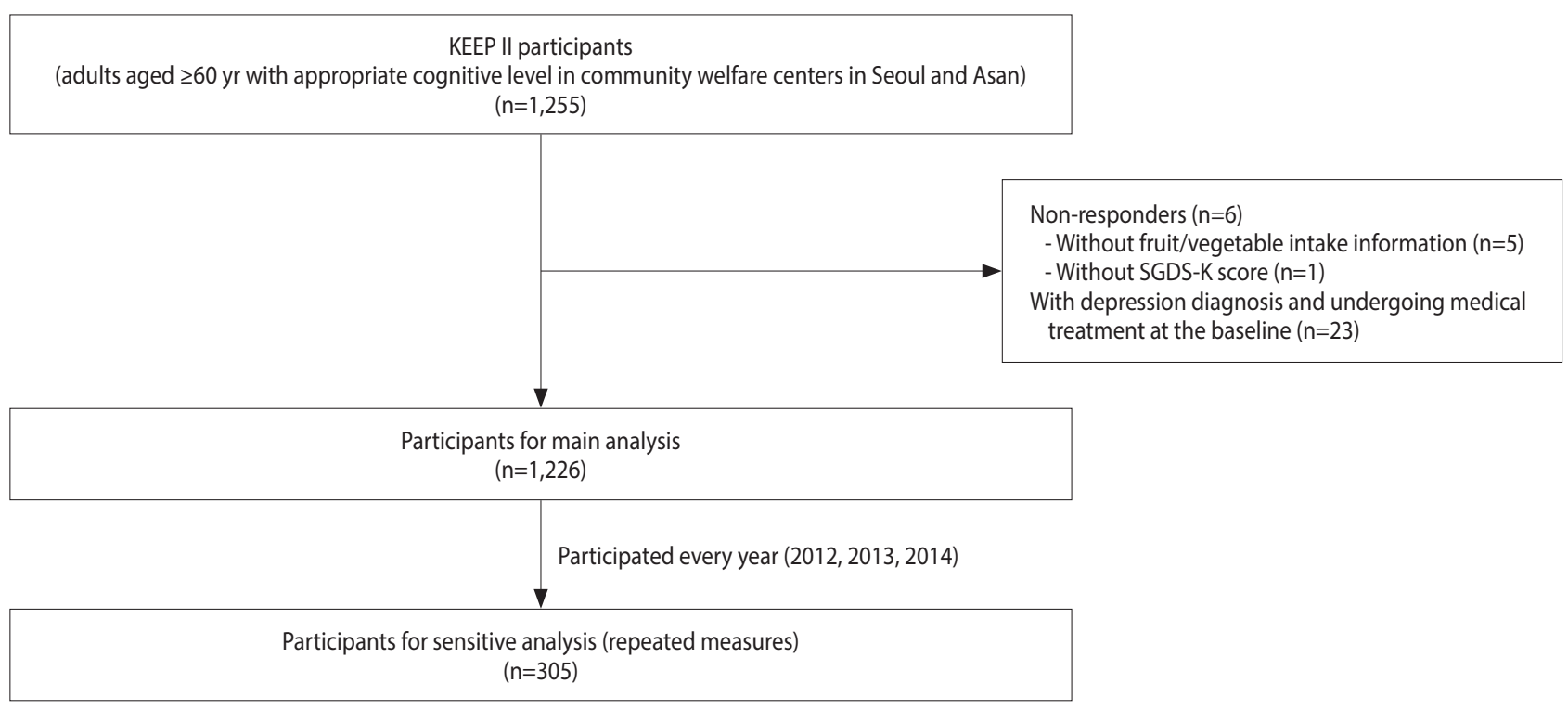

Figure 1. Selection of participants. KEEP, Korean Elderly Environmental Panel; SGDS-K, Korean version of the Short form Geriatric Depression Scale. 
the response "yes" or "no," with "yes" counted as 1 and "no" counted as 0 to yield a range of total score from 0 to 15 . As the SGDS-K score increases, people experience more depressive symptoms. A score of 8 was used as the cut-off point for the diagnosis of depression. Previous studies have established that the SGDS-K has high validity for the diagnosis of depression [20].

\section{Dietary assessment}

The dietary assessment was performed using a self-reporting method of responses to simple questions regarding fruit and vegetable consumption: "How often do you usually eat fruit? Fruits include fresh, dried, canned, and frozen fruit." and "How often do you usually eat vegetables? Vegetables include fresh, dried, canned, and frozen vegetables." The following choices were provided: (a) never, (b) less than 1 time/wk, (c) 1-3 times/wk, (d) 4-6 times/wk, (e) daily, and (f) do not know. The data signified the frequency, not the quantity, of consumption.

More detailed dietary information including the intake of each food item in grams, total energy intake, and daily nutrient intake were investigated for the year 2012 by using a semiquantitative food frequency questionnaire (FFQ) to assess the frequency of consumption ( 9 categories from rarely eaten to eaten more than 3 times/d) and portion size (categorized as small, average, or large) of 118 food items [21,22]. The amount of each food item was transformed into grams using the Computer Aided Nutritional Analysis Program for Professionals (CAN-pro 4.0, Korean Society of Nutrition, Seoul, Korea). However, because only 385 participants participated in the FFQ and the FFQ was conducted only in 2012, we decided to use the simple questions mentioned above.

\section{Other covariates}

Age was categorized into three groups: 60-69 years, 70-79 years, and $\geq 80$ years. Education level was categorized into three levels (detailed information provided in Supplementary Material 2). Allowance was categorized as $<30.00,30.00-69.99$, and $\geq 70.00$ units of 10,000 Korean won (KRW) per month (approximately US $\$ 10$ ). Only current behavior was considered to assess smoking status and alcohol consumption. Household type was categorized as living alone or living with family, which refers to living with spouse, sons or daughters, or others. The presence or absence of underlying diseases was assessed. Disease status refers to a history of admission/surgery/emergency room visit within 1 year or a visit to a doctor without admission within 2 weeks. We used the short form of the International Physical Activity Questionnaire to assess physical activity levels. Physical activity was coded as $<600$ or $\geq 600$ metabolic equivalents of task (MET) min/wk, with MET measured as energy expenditure in calories per kilogram of body weight for 1 minute $(\mathrm{kcal} / \mathrm{min} / \mathrm{kg}$ ) (Supplementary Material 2). Body mass index (BMI) was calculated as weight divided by height squared and categorized as $<23.0 \mathrm{~kg} / \mathrm{m}^{2}, 23.0-24.9 \mathrm{~kg} / \mathrm{m}^{2}$, and $\geq 25.0 \mathrm{~kg} / \mathrm{m}^{2}$, with $\geq 23.0 \mathrm{~kg} / \mathrm{m}^{2}$ classified as overweight according to the Korean Society for the Study of Obesity [23]. According to the Korean Society for the Study of Obesity, $\geq 25.0 \mathrm{~kg} / \mathrm{m}^{2}$ is classified as obese, although this value is classified as overweight by the World Health Organization $[23,24]$.

\section{Statistical analysis}

The t-test was used to compare continuous variables such as age and total energy intake in participants with depression (SGDS-K $\geq 8$ ) and without depression (SGDS-K $<8$ ), whereas chi-square tests were used to compare categorical variables such as sex, age category, education, allowance, smoking status, alcohol consumption status, household type, disease status, physical activity, BMI category, weekly fruit intake, and weekly vegetable intake.

The SGDS-K was evaluated as both a continuous and categorical variable. The SGDS-K score as a continuous variable refers to the level of depression, and as a categorical variable, it refers to depression status (SGDS-K $\geq 8$ or $<8$ ). The association between the level of depression and the frequency of fruit or vegetable consumption was also estimated. Multivariate linear regression was used to estimate $\beta, 95 \%$ confidence interval (CI), and $\mathrm{p}$-value for SGDS-K scores (continuous variable). Multivariate logistic regression was used to estimate odds ratios (ORs), 95\% CIs, and p-values for depression status (SGDS $\geq 8$ or $<8$ ). The analyses were performed using three models: a crude model, an age- and sexadjusted model, and a multiple covariate-adjusted model. The confounding factors used in the multiple covariate-adjusted models were sex, age category (the age- and sex-adjusted model used age as a continuous variable), education, allowance, smoking status, alcohol consumption status, household type, disease status, physical activity, and BMI.

For sensitivity analysis, reciprocal adjustment for weekly vegetable intake and weekly fruit intake was performed in multiple covariate-adjusted models. Also, we performed sensitivity analysis by combining "never" and "less than 1 time" together as the sample sizes of these categories were small. Furthermore, generalized linear mixed-effects models were used for repeated measures in the subset of subjects who repeatedly participated in the KEEP survey 3 times. Linear mixed-effects models were also constructed as crude, age- and sex-adjusted, and multiple covariate-adjusted models.

To assess dose-response relationships, we regarded the frequency of weekly fruit and vegetable intake as a continuous variable; "never," "less than 1 time/wk," "1-3 times/wk," "4-6 times/wk," and "daily" were converted into 0 times/wk, 0.5 times/wk, 2 times/wk, 5 times/wk, and 7 times/wk, respectively, as these values are the average value of each category. We then assessed the dose-dependent relationship of the level of depression, that is, SGDS-K score (continuous variable), and depression status (SGDS-K $<8$ or $\geq 8$ ) with fruit and vegetable consumption using linear regression and logistic regression analyses, respectively. $\mathrm{R}$ version 4.0.3. (https:// cran.r-project.org/) and SPSS version 25.0 (IBM Corp., Armonk, NY, USA) were used for the statistical analysis. In the present study, a p-value $<0.05$ was considered statistically significant. 
Table 1. General characteristics of study participants

\begin{tabular}{|c|c|c|c|c|}
\hline Characteristics & No depression & Depression ${ }^{1}$ & Total & $p$-value ${ }^{2}$ \\
\hline Total & $985(80.3)$ & $241(19.7)$ & $1,226(100)$ & 0.001 \\
\hline \multicolumn{5}{|l|}{ Sex } \\
\hline Male & $324(32.9)$ & $53(22.0)$ & $377(30.7)$ & \\
\hline Female & $661(67.1)$ & $188(78.0)$ & $849(69.2)$ & \\
\hline Age (yr) & $73.94 \pm 6.04$ & $75.53 \pm 6.39$ & $74.25 \pm 6.14$ & $<0.001$ \\
\hline $60-69$ & $234(23.7)$ & $38(15.8)$ & $272(22.2)$ & 0.022 \\
\hline $70-79$ & $558(56.6)$ & $146(60.6)$ & $704(57.4)$ & \\
\hline$\geq 80$ & $193(19.6)$ & $57(23.6)$ & $250(20.4)$ & \\
\hline Education & & & & 0.001 \\
\hline$<$ Middle school graduation & $645(65.5)$ & $186(77.2)$ & $831(67.8)$ & \\
\hline $\begin{array}{l}\text { Middle school graduation to two-year } \\
\text { college graduation }\end{array}$ & $268(27.2)$ & $49(20.3)$ & $317(25.8)$ & \\
\hline$\geq$ Four-year university dropout & $72(7.3)$ & $6(2.4)$ & $78(6.4)$ & \\
\hline Allowance $\left(10^{4} \mathrm{KRW} / \mathrm{mo}\right)^{3}$ & 950 & 227 & 1,177 & 0.129 \\
\hline$<30.00$ & $678(71.4)$ & $177(78.0)$ & $855(72.6)$ & \\
\hline $30.00-69.99$ & $196(20.6)$ & $37(16.0)$ & $233(19.8)$ & \\
\hline$\geq 70.00$ & $76(8.0)$ & $13(6.0)$ & $89(7.6)$ & \\
\hline Smoking & $48(4.9)$ & $13(5.4)$ & $61(5.0)$ & 0.739 \\
\hline Alcohol drinking & $206(20.9)$ & $44(18.3)$ & $250(20.4)$ & 0.359 \\
\hline Household type & & & & $<0.001$ \\
\hline Living alone & $289(29.3)$ & $118(49.0)$ & $407(33.2)$ & \\
\hline Living with family & $696(70.7)$ & $123(51.0)$ & $819(66.8)$ & \\
\hline Disease status (yes) ${ }^{4}$ & $456(46.3)$ & $146(60.6)$ & $602(49.1)$ & $<0.001$ \\
\hline Physical activity (MET min/wk) & 977 & 239 & 1,216 & 0.003 \\
\hline$<600$ & $591(60.5)$ & $169(70.7)$ & $760(62.5)$ & \\
\hline$\geq 600$ & $386(39.5)$ & $70(29.3)$ & $456(37.5)$ & \\
\hline Body mass index $\left(\mathrm{kg} / \mathrm{m}^{2}\right)$ & 963 & 234 & 1,197 & 0.681 \\
\hline$<23.0$ & $409(42.5)$ & $93(39.7)$ & $502(41.9)$ & \\
\hline $23.0-24.9$ & $243(25.2)$ & $59(25.2)$ & $302(25.2)$ & \\
\hline$\geq 25.0$ & $311(32.2)$ & $82(35.0)$ & $393(32.8)$ & \\
\hline Total energy intake, $n=385(\mathrm{kcal} / \mathrm{d})$ & $1,804.80 \pm 352.37$ & $1,470.37 \pm 911.29$ & $1,707.51 \pm 881.94$ & 0.001 \\
\hline \multicolumn{5}{|l|}{ Weekly intake (times/wk) } \\
\hline Fruit & & & & $<0.001$ \\
\hline Never & $95(9.6)$ & 48 (19.9) & $143(11.7)$ & \\
\hline$<1$ & $120(12.2)$ & $34(14.1)$ & $154(12.6)$ & \\
\hline $1-3$ & $281(28.5)$ & 81 (33.6) & $362(29.5)$ & \\
\hline $4-6$ & $158(16.0)$ & $22(9.1)$ & $180(14.7)$ & \\
\hline Everyday & 331 (33.6) & $56(23.2)$ & 387 (31.6) & \\
\hline Vegetable & & & & 0.228 \\
\hline Never & $32(3.2)$ & $14(5.8)$ & $46(3.7)$ & \\
\hline$<1$ & $25(2.5)$ & $3(1.2)$ & $28(2.3)$ & \\
\hline $1-3$ & 107 (10.9) & $29(12.0)$ & $136(11.1)$ & \\
\hline $4-6$ & $103(10.4)$ & $21(8.7)$ & $124(10.1)$ & \\
\hline Everyday & 718 (72.9) & $174(72.2)$ & 892 (72.7) & \\
\hline
\end{tabular}

Values are presented as number (\%) or mean \pm standard deviation.

SGDS-K, Korean version of the Short form Geriatric Depression Scale; KRW, Korean won; MET, metabolic equivalents of task. 'SGDS-K $\geq 8$.

${ }^{2}$ To derive $\mathrm{p}$-values, $\mathrm{t}$-test was used for continuous variables, and chi-square test was used for categorical variables.

${ }^{3} 10,000$ KRW (roughly, US\$10).

${ }^{4} \mathrm{Admission} /$ surgery/emergency room visit within 1 year, visit to a doctor without admission within 2 weeks. 


\section{Ethics statement}

All participants provided written informed consent, and the study protocol was approved by the Institutional Review Board (IRB) of the Seoul National University College of Medicine (IRB No. 0804-045-241).

\section{RESULTS}

The baseline characteristics of the participants are shown in Table 1 . Among the 1,226 participants, $19.7 \%$ had depression. Compared to the participants without depression, the participants with depression were more likely to be female (78.0 vs. 67.1\%) and older (75.53 vs. 73.94 years) and to have a lower educational level (less than middle school graduation: 77.2 vs. $65.5 \%)$, live alone (49.0 vs. $29.3 \%$ ), and have an underlying disease (60.6 vs. $46.3 \%)$. Participants with depression were less likely to be physically active $(<600$ MET min/wk: 70.7 vs. $60.5 \%$ ), consume adequate calories $(1,470.37 \mathrm{vs} .1,804.80 \mathrm{kcal} / \mathrm{d})$, and consume fruits frequently (nev- er: 19.9 vs. $9.6 \%$; less than once/wk: 14.1 vs. $12.2 \%$; $1-3$ times/wk: 33.6 vs. $28.5 \%$; $4-6$ times/wk: 9.1 vs. $16.0 \%$; and daily: 23.2 vs. $33.6 \%)$. However, the current study did not observe any significant differences between the groups with and without depression with regard to smoking status, alcohol consumption, BMI, and weekly vegetable intake (Table 1 ).

The association between weekly fruit intake, SGDS-K score, and depression status is shown in Table 2. The SGDS-K score was significantly and negatively associated with the frequency of fruit consumption in the crude, age- and sex-adjusted, and multiple covariate-adjusted models. The effect size of the association between fruit consumption and SGDS-K score increased with an increase in the frequency of fruit consumption. For instance, the $\beta$ estimation for the association between fruit consumption frequency and SGDS-K score in the subjects who consumed fruits 1-3 times/wk was -0.17 (95\% CI, -0.27 to -0.07), compared to the group with no fruit consumption. Fruit consumption at a frequency of 4-6 times/wk showed a greater association with SGDS-

Table 2. Cross-sectional multiple linear regression models for the association of weekly fruit/vegetable intake with SGDS-K and depression status $(n=1,226)$

\begin{tabular}{|c|c|c|c|c|c|c|}
\hline Weekly intake & Crude model & p-value & $\begin{array}{c}\text { Age- and } \\
\text { sex-adjusted model }\end{array}$ & $\mathrm{p}$-value & 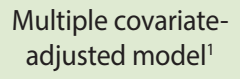 & $\mathrm{p}$-value \\
\hline \multicolumn{7}{|c|}{ Fruit (times/wk) } \\
\hline \multicolumn{7}{|c|}{ SGDS-K, $\beta(95 \%$ CI) } \\
\hline Never & 0.00 & & 0.00 & & 0.00 & \\
\hline$<1$ & $-0.17(-0.27,-0.07)$ & 0.001 & $-0.17(-0.28,-0.07)$ & $<0.001$ & $-0.17(-0.28,-0.05)$ & 0.005 \\
\hline $1-3$ & $-0.26(-0.35,-0.17)$ & $<0.001$ & $-0.24(-0.33,-0.16)$ & $<0.001$ & $-0.17(-0.27,-0.07)$ & 0.001 \\
\hline $4-6$ & $-0.61(-0.72,-0.50)$ & $<0.001$ & $-0.55(-0.66,-0.44)$ & $<0.001$ & $-0.42(-0.54,-0.29)$ & $<0.001$ \\
\hline Everyday & $-0.53(-0.62,-0.44)$ & $<0.001$ & $-0.50(-0.59,-0.41)$ & $<0.001$ & $-0.33(-0.44,-0.21)$ & $<0.001$ \\
\hline \multicolumn{7}{|c|}{ Depression status, OR ( $95 \% \mathrm{Cl})$} \\
\hline Never & 1.00 (reference) & & 1.00 (reference) & & 1.00 (reference) & \\
\hline$<1$ & $0.56(0.33,0.94)$ & 0.028 & $0.55(0.33,0.93)$ & 0.025 & $0.65(0.39,1.07)$ & 0.087 \\
\hline $1-3$ & $0.57(0.37,0.87)$ & 0.010 & $0.58(0.38,0.90)$ & 0.014 & $0.77(0.51,1.16)$ & 0.205 \\
\hline $4-6$ & $0.28(0.16,0.48)$ & $<0.001$ & $0.30(0.17,0.53)$ & $<0.001$ & $0.42(0.23,0.75)$ & 0.003 \\
\hline Everyday & $0.33(0.21,0.52)$ & $<0.001$ & $0.36(0.23,0.57)$ & $<0.001$ & $0.55(0.34,0.89)$ & 0.015 \\
\hline \multicolumn{7}{|c|}{ Vegetable (times/wk) } \\
\hline \multicolumn{7}{|c|}{ SGDS-K, $\beta(95 \%$ CI) } \\
\hline Never & 0.00 & & 0.00 & & 0.00 & \\
\hline$<1$ & $-0.68(-0.95,-0.43)$ & $<0.001$ & $-0.70(-0.96,-0.44)$ & $<0.001$ & $-0.86(-1.18,-0.55)$ & $<0.001$ \\
\hline $1-3$ & $-0.20(-0.35,-0.05)$ & 0.010 & $-0.14(-0.29,0.01)$ & 0.062 & $-0.18(-0.35,-0.01)$ & 0.038 \\
\hline $4-6$ & $-0.53(-0.69,-0.36)$ & $<0.001$ & $-0.43(-0.59,-0.27)$ & $<0.001$ & $-0.36(-0.53,-0.18)$ & $<0.001$ \\
\hline Everyday & $-0.30(-0.43,-0.17)$ & $<0.001$ & $-0.22(-0.35,-0.08)$ & 0.001 & $-0.15(-0.29,0.00)$ & 0.048 \\
\hline \multicolumn{7}{|c|}{ Depression status, OR ( $95 \% \mathrm{Cl})$} \\
\hline Never & 1.00 (reference) & & 1.00 (reference) & & 1.00 (reference) & \\
\hline$<1$ & $0.27(0.07,1.06)$ & 0.061 & $0.26(0.07,1.02)$ & 0.054 & $0.22(0.04,1.10)$ & 0.065 \\
\hline $1-3$ & $0.62(0.29,1.31)$ & 0.211 & $0.69(0.32,1.48)$ & 0.339 & $0.62(0.26,1.47)$ & 0.282 \\
\hline $4-6$ & $0.47(0.21,1.02)$ & 0.056 & $0.55(0.25,1.23)$ & 0.147 & $0.59(0.25,1.43)$ & 0.245 \\
\hline Everyday & $0.55(0.29,1.06)$ & 0.075 & $0.65(0.34,1.26)$ & 0.204 & $0.70(0.33,1.48)$ & 0.353 \\
\hline
\end{tabular}

SGDS-K, Korean version of the Short form Geriatric Depression Scale; OR, odds ratio; Cl, confidence interval; KRW, Korean won; MET, metabolic equivalents of task.

${ }^{1}$ Adjusted for age, sex, education, allowance (10,000 KRW/mo), smoking status, alcohol consumption status, household type, disease status, physical activity (MET min/wk), and body mass index. 
K scores ( $\beta,-0.42 ; 95 \% \mathrm{CI},-0.54$ to -0.29$)$. The OR of depression in the elderly subjects who consumed fruits $4-6$ times/wk was 0.42 (95\% CI, 0.23 to 0.75 ), compared to the group with no fruit consumption, whereas the OR pertaining to the group with daily fruit consumption was greater (OR, $0.55 ; 95 \% \mathrm{CI}, 0.34$ to 0.89 ) (Table 2).

The associations of weekly vegetable intake with the SGDS-K score and depression status are shown in Table 2. The SGDS-K scores were negatively associated with vegetable consumption frequency in the subjects who consumed vegetables $4-6$ times/wk ( $\beta,-0.36 ; 95 \% \mathrm{CI},-0.53$ to -0.18 ), compared to the subjects who did not consume vegetables. The association between vegetable consumption and depression status was not significant (Table 2).

\section{Sensitivity analysis}

Reciprocal adjustment for fruit or vegetable consumption frequency did not affect the overall results (Supplementary Materials
3 and 4). Furthermore, as we grouped "never" and "less than once/ week" together (Supplementary Materials 5 and 6), we found that the trends of results were not different from the main analyses. The present study performed additional analyses using generalized linear mixed-effects models to analyze the repeated measures pertaining to the subjects who participated in the survey three consecutive times $(n=305)$. The risk of depression decreased with an increase in the weekly frequency of fruit consumption in a dosedependent manner. In the multiple covariate-adjusted model, the ORs for depression were 1.34 (95\% CI, 0.48 to 3.71), 0.66 (95\% CI, 0.27 to 1.60 ), 0.53 (95\% CI, 0.18 to 1.56 ), and 0.40 (95\% CI, 0.16 to 1.02) at weekly frequencies of less than 1 time/wk, $1-3$ times/wk, 4-6 times/wk, and daily. However, significant results were observed only in relation to weekly fruit consumption of 4-6 times/wk in the crude model and the daily fruit consumption in the crude and age- and sex-adjusted models (Table 3). There was no significant association between vegetable consumption and the level of de-

Table 3. Generalized linear mixed-effects models for the association of weekly fruit/vegetable intake with SGDS-K and depression status ( $n=305 ; 915$ observations)

\begin{tabular}{|c|c|c|c|c|c|c|}
\hline Weekly intake & Crude model & $p$-value & $\begin{array}{c}\text { Age- and } \\
\text { sex-adjusted model }\end{array}$ & p-value & $\begin{array}{l}\text { Multiple covariate- } \\
\text { adjusted model }^{1}\end{array}$ & $\mathrm{p}$-value \\
\hline \multicolumn{7}{|c|}{ Fruit (times/wk) } \\
\hline \multicolumn{7}{|c|}{ SGDS-K, $\beta(95 \%$ CI) } \\
\hline Never & 0.00 & & 0.00 & & 0.00 & \\
\hline$<1$ & $0.05(-0.12,0.22)$ & 0.566 & $0.05(-0.11,0.22)$ & 0.547 & $0.08(-0.09,0.26)$ & 0.353 \\
\hline $1-3$ & $-0.09(-0.24,0.06)$ & 0.218 & $-0.09(-0.24,0.05)$ & 0.204 & $-0.09(-0.24,0.07)$ & 0.205 \\
\hline $4-6$ & $-0.14(-0.32,0.04)$ & 0.132 & $-0.14(-0.32,0.05)$ & 0.141 & $-0.13(-0.32,0.06)$ & 0.167 \\
\hline Everyday & $-0.12(-0.29,0.05)$ & 0.167 & $-0.11(-0.28,0.05)$ & 0.187 & $-0.10(-0.28,0.07)$ & 0.244 \\
\hline \multicolumn{7}{|c|}{ Depression status, OR (95\% Cl) } \\
\hline Never & 1.00 (reference) & & 1.00 (reference) & & 1.00 (reference) & \\
\hline$<1$ & $1.33(0.47,3.72)$ & 0.591 & $1.41(0.51,3.92)$ & 0.511 & $1.34(0.48,3.71)$ & 0.574 \\
\hline $1-3$ & $0.43(0.17,1.08)$ & 0.071 & $0.45(0.18,1.13)$ & 0.089 & $0.66(0.27,1.60)$ & 0.356 \\
\hline $4-6$ & $0.32(0.10,0.95)$ & 0.041 & $0.36(0.12,1.08)$ & 0.068 & $0.53(0.18,1.56)$ & 0.248 \\
\hline Everyday & $0.21(0.08,0.53)$ & 0.001 & $0.25(0.10,0.65)$ & 0.004 & $0.40(0.16,1.02)$ & 0.056 \\
\hline \multicolumn{7}{|c|}{ Vegetables (times/wk) } \\
\hline \multicolumn{7}{|c|}{ SGDS-K, $\beta(95 \% \mathrm{CI})$} \\
\hline Never & 0.00 & & 0.00 & & 0.00 & \\
\hline$<1$ & $0.18(-0.22,0.59)$ & 0.369 & $0.17(-0.22,0.57)$ & 0.396 & $0.11(-0.31,0.52)$ & 0.613 \\
\hline $1-3$ & $0.10(-0.25,0.47)$ & 0.569 & $0.10(-0.25,0.45)$ & 0.566 & $-0.03(-0.40,0.33)$ & 0.856 \\
\hline $4-6$ & $-0.02(-0.38,0.36)$ & 0.922 & $-0.01(-0.38,0.35)$ & 0.942 & $-0.18(-0.56,0.20)$ & 0.357 \\
\hline Everyday & $0.11(-0.22,0.46)$ & 0.512 & $0.12(-0.22,0.45)$ & 0.498 & $0.06(-0.29,0.41)$ & 0.745 \\
\hline \multicolumn{7}{|c|}{ Depression status, OR ( $95 \% \mathrm{Cl})$} \\
\hline Never & 1.00 (reference) & & 1.00 (reference) & & 1.00 (reference) & \\
\hline$<1$ & $3.71(0.30,46.42)$ & 0.308 & $3.43(0.29,40.96)$ & 0.331 & $1.76(0.16,19.16)$ & 0.644 \\
\hline $1-3$ & $1.90(0.20,17.66)$ & 0.572 & $1.97(0.22,17.50)$ & 0.544 & $0.96(0.12,7.91)$ & 0.968 \\
\hline $4-6$ & $1.18(0.12,11.87)$ & 0.885 & $1.29(0.13,12.41)$ & 0.824 & $0.79(0.09,7.21)$ & 0.832 \\
\hline Everyday & $1.83(0.22,14.98)$ & 0.572 & $2.01(0.26,15.84)$ & 0.506 & $1.58(0.22,11.29)$ & 0.648 \\
\hline
\end{tabular}

SGDS-K, Korean version of the Short form Geriatric Depression Scale; OR, odds ratio; Cl, confidence interval; KRW, Korean won; MET, metabolic equivalents of task.

'Adjusted for age, sex, education, allowance (10,000 KRW/mo), smoking status, alcohol consumption status, household type, disease status, physical activity (MET min/wk), and body mass index. 
Yuk Y et al. : Fruit and vegetable consumption and depression

Table 4. The dose-dependent relationship between the weekly fruit/vegetable intake and SGDS-K ( $\beta$ ) and depression status $(\mathrm{OR})^{1}$

\begin{tabular}{lcrcr}
\hline Variables & $\beta(95 \% \mathrm{Cl})$ & $\mathrm{p}$-value & OR $(95 \% \mathrm{Cl})$ & $\mathrm{p}$-value \\
\hline Cross-sectional multiple linear regression model $(\mathrm{n}=1,226)$ & & & & \\
$\quad$ Weekly fruit intake & $-0.04(-0.06,-0.03)$ & $<0.001$ & $0.90(0.84,0.97)$ & 0.004 \\
Weekly vegetable intake & $0.01(-0.00,0.03)$ & 0.151 & $1.02(0.94,1.10)$ & 0.672 \\
Generalized linear mixed-effects model $(\mathrm{n}=305 ; 915$ observations) & $-0.04(-0.06,-0.03)$ & $<0.001$ & $0.87(0.78,0.96)$ & 0.006 \\
Weekly fruit intake & $0.00(-0.01,0.02)$ & 0.631 & $1.07(0.94,1.22)$ & 0.330 \\
Weekly vegetable intake & & & \\
\hline
\end{tabular}

SGDS-K, Korean version of the Short form Geriatric Depression Scale; OR, odds ratio; Cl, confidence interval; KRW, Korean won; MET, metabolic equivalents of task.

${ }^{1}$ Adjusted for age, sex, education, allowance (10,000 KRW/mo), smoking status, alcohol consumption status, household type, disease status, physical activity (MET min/wk), and body mass index.

pression or depression status with regard to repeated measures (Table 3).

\section{Dose-dependent relationship}

Regarding the dose-dependent relationship, the SGDS-K scores had a negative linear relationship with the frequency of weekly fruit consumption ( $\beta,-0.04 ; 95 \% \mathrm{CI},-0.06$ to -0.03$)$. This pattern was consistent in the linear mixed-effects model $(\beta,-0.04 ; 95 \% \mathrm{CI}$, -0.06 to -0.03$)$. The greater the frequency of fruit consumption, the lower the risk of depression in both the cross-sectional model (OR, 0.90; 95\% CI, 0.84 to 0.97) and repeated measures (OR, 0.87; $95 \% \mathrm{CI}, 0.78$ to 0.96 ), when the frequency of fruit consumption was considered as a continuous variable. The linearity was not significant in the case of vegetable consumption (Table 4).

\section{DISCUSSION}

The current study showed that fruit consumption was inversely associated with the level of depression in a dose-dependent manner. Although the level of depression did not have a dose-dependent relationship with vegetable consumption frequency, vegetable consumption significantly lowered the SGDS-K scores compared to no vegetable consumption.

Previous studies have reported inconsistent results regarding the association between the level of depression and fruit or vegetable consumption. Nevertheless, a couple of studies have reported that fruit consumption is more inversely associated with depressive symptoms than vegetable consumption, which is consistent with the current results $[6,8]$. However, certain studies have reported significant associations in relation to both fruit and vegetable consumption $[10,11]$, whereas other studies did not detect such significant associations [7,9].

The frequency of vegetable consumption did not show a dosedependent relationship with the level of depressive symptoms and was not significantly associated with depression status. This finding could be attributed to the fact that more than $70 \%$ of the participants responded that they consumed vegetables every day, whereas less than $30 \%$ of the participants with depression responded with other choices. Moreover, the distribution of vegetable intake frequency in the subjects with and without depression was similar, which is mainly attributable to the fact that most Korean foods are vegetable-based and most Koreans consume vegetables every day. A basic Korean meal includes rice, soup, and kimchi, which are vegetable-based foods, in addition to soybean or vegetable-based dishes [25]. The availability of more comprehensive data is needed in future studies. For instance, certain cross-sectional studies have shown a dose-dependent relationship between the amount of fruit and vegetable intake and the level of depression $[5,26]$.

\section{Plausible mechanisms}

Fruits and vegetables contain several nutrients that are known to prevent depressive symptoms. Defects in antioxidant defense systems are associated with a high incidence of depression. This is because depression increases the production of proinflammatory cytokines, which induce reactive oxygen species, which then trigger the lipid peroxidation process, compromising the brain $[27,28]$. Hence, antioxidants such as vitamins $\mathrm{C}, \mathrm{E}$, and $\beta$-carotene play an important role [3,27]. For example, vitamin E prevents peroxidation of $\omega 3$ polyunsaturated fatty acid [25]. Nutrients such as folate are essential for the biosynthesis of neurotransmitters such as serotonin, which stabilizes mood, as folate acts as a methyl donor, which is essential for the conversion of homocysteine to methionine by methylation [29]. Thus, folate deficiency is associated with a high risk of depression as neurotransmitters such as serotonin decrease [30]. Vitamin B6 is also known to mitigate the symptoms of hormone-related depression, as vitamin B6 is also involved in the synthesis of neurotransmitters that regulate emotions [31].

Conversely, a plausible explanation for the inverse association between depression and fruit consumption, in contrast to vegetable consumption, is that fruits can substitute for sugar-rich desserts. As sugar consumption has been associated with a higher incidence of depression in six countries [32], eating fruits instead of a sugarrich dessert may have a more desirable effect in preventing depressive symptoms. Additionally, several antioxidative nutrients, such as tangeretin, nobiletin, rutin, and resveratrol, are present only in fruits and not in vegetables [31]. 


\section{Strengths}

First, the data were analyzed for both the level and risk of depression. Most previous studies focused on the OR of depression, whereas the present study also presented the level of depressive symptoms using the SGDS-K scores. Second, unlike most crosssectional studies, the present study conducted a longitudinal analysis using a generalized linear mixed-effects model. Lastly, to the best of our knowledge, this is the first study to analyze the associations between the level of depressive symptoms, which is explained by the SGDS-K score, and fruit and vegetable consumption in elderly Korean people exclusively, although a few studies have involved Korean adults [12,13] and adolescents [14,15]. Ji et al. [12] included female subjects below 70 years of age, which may not represent the elderly Korean population. A study by Ju \& Park [13] covered both the sex and age of elderly subjects; however, vegetable and fruit consumption were not analyzed separately.

\section{Limitations}

First, only the frequency of dietary intake was included in the analyses. Hence, data regarding the quantity, quality, cooking methods, nutrient density, and the proportion of fruits and vegetables in the total dietary intake were unavailable. In addition, it was not clear if fruit juice or kimchi was included in the fruit or vegetable consumption, considering the character of the question in the questionnaire. However, as the current study involved elderly subjects and considering that the KEEP-II study included numerous questions that were answered repeatedly every year, enquiries regarding the usual dietary behavior, such as the weekly frequency of consumption, may be more realistic and intuitive for the participants than enquiries about the exact quantity of each food item, which results in higher data reliability.

Second, depression was not assessed using clinical diagnoses; instead, the SGDS-K was used as a screening tool. However, considering that the subjects were elderly, feeling depressed may not warrant a visit to the doctor, as they are reluctant to burden their families and do not perceive depression as a serious condition. Accordingly, SGDS-K may be more inclusive.

Third, this study used a cross-sectional analysis. Hence, the causality of fruit and vegetable consumption with respect to the level of depression could not be proven. As depression can alter dietary style, reverse causation cannot be excluded in the correlation between the variables. Further longitudinal or cohort studies are needed to confirm the causality.

Fourth, more frequent fruit or vegetable intake, such as twice or thrice a day, was not considered. For example, elderly Korean individuals often eat kimchi at every meal. Thus, for future studies, a clearer representation of the food category is required.

Lastly, because the survey used a self-reporting method, recall bias cannot be excluded, especially in older adults. Furthermore, there may be residual confounding factors, such as total energy intake, which was obtained in only a small number of people who participated in the FFQ in $2012(\mathrm{n}=385)$; thus, BMI was used instead. Cognitive status was not considered, as the participants of the KEEP survey were required to have an adequate cognitive level. Therefore, the current study is only applicable to fully cognitive elderly individuals with typical Korean dietary behavior.

In conclusion, the present study implies that the level of depression, which is explained by the SGDS-K score, is inversely associated with the frequency of fruit intake in a dose-dependent manner. Vegetable consumption did not display a dose-dependent relationship with depression level. However, SGDS-K scores were significantly negatively associated with vegetable intake compared to no vegetable consumption. Further studies are required to elucidate the underlying mechanisms and to identify the causality between the frequency of fruit and vegetable intake and the level of depression.

\section{SUPPLEMENTARY MATERIALS}

Supplementary materials are available at https://www.e-epih.org/.

\section{CONFLICT OF INTEREST}

The authors have no conflicts of interest to declare for this study.

\section{FUNDING}

This study was supported by the Environmental Exposures and Health Effects in Elderly Population Programme of the National Institute of Environmental Research, Ministry of Environment (2012KOELPO1), Korea.

\section{ACKNOWLEDGEMENTS}

None.

\section{AUTHOR CONTRIBUTIONS}

Conceptualization: YY, YJC, YCH. Formal analysis: YY, YJC, YJ. Funding acquisition: YCH. Writing - original draft: YY. Writing - review \& editing: YY, YJC, YCH, CRH, YJ.

\section{ORCID}

Youjeong Yuk: https://orcid.org/0000-0002-5948-5968; ChaeRin Han: https://orcid.org/0000-0003-2675-5535; Yoonyoung Jang: https://orcid.org/0000-0002-8161-9950; Yun-Chul Hong: https:// orcid.org/0000-0001-9010-7271; Yoon-Jung Choi: https://orcid. org/0000-0001-8168-0228

\section{REFERENCES}

1. Hidaka BH. Depression as a disease of modernity: explanations for increasing prevalence. J Affect Disord 2012;140:205-214.

2. Moussavi S, Chatterji S, Verdes E, Tandon A, Patel V, Ustun B. Depression, chronic diseases, and decrements in health: results 
from the World Health Surveys. Lancet 2007;370:851-858.

3. Saghafian F, Malmir H, Saneei P, Milajerdi A, Larijani B, Esmaillzadeh A. Fruit and vegetable consumption and risk of depression: accumulative evidence from an updated systematic review and metaanalysis of epidemiological studies. Br J Nutr 2018;119:1087-1101.

4. Blanchflower DG, Oswald AJ, Stewart-Brown S. Is psychological well-being linked to the consumption of fruit and vegetables? Soc Indic Res 2013;114:785-801.

5. Bishwajit G, O'Leary DP, Ghosh S, Sanni Y, Shangfeng T, Zhanchun F. Association between depression and fruit and vegetable consumption among adults in South Asia. BMC Psychiatry 2017;17:15.

6. Mihrshahi S, Dobson AJ, Mishra GD. Fruit and vegetable consumption and prevalence and incidence of depressive symptoms in mid-age women: results from the Australian longitudinal study on women's health. Eur J Clin Nutr 2015;69:585-591.

7. Chi SH, Wang JY, Tsai AC. Combined association of leisure-time physical activity and fruit and vegetable consumption with depressive symptoms in older Taiwanese: results of a national cohort study. Geriatr Gerontol Int 2016;16:244-251.

8. Sánchez-Villegas A, Delgado-Rodríguez M, Alonso A, Schlatter J, Lahortiga F, Serra Majem L, et al. Association of the Mediterranean dietary pattern with the incidence of depression: the Seguimiento Universidad de Navarra/University of Navarra follow-up (SUN) cohort. Arch Gen Psychiatry 2009;66:1090-1098.

9. Hintikka J, Tolmunen T, Honkalampi K, Haatainen K, KoivumaaHonkanen H, Tanskanen A, et al. Daily tea drinking is associated with a low level of depressive symptoms in the Finnish general population. Eur J Epidemiol 2005;20:359-363.

10. Akbaraly TN, Sabia S, Shipley MJ, Batty GD, Kivimaki M. Adherence to healthy dietary guidelines and future depressive symptoms: evidence for sex differentials in the Whitehall II study. Am J Clin Nutr 2013;97:419-427.

11. Konttinen H, Männistö S, Sarlio-Lähteenkorva S, Silventoinen K, Haukkala A. Emotional eating, depressive symptoms and self-reported food consumption. A population-based study. Appetite 2010;54:473-479.

12. Ji J, Kim Y, Shin WK. Association between fruit and vegetable consumption and mental health according to physical activity among Korean adult women-Korea National Health and Nutrition Examination Survey 2014-2016. J Korean Soc Food Cult 2020; 35:107-116 (Korean).

13. Ju SY, Park YK. Low fruit and vegetable intake is associated with depression among Korean adults in data from the 2014 Korea National Health and Nutrition Examination Survey. J Health Popul Nutr 2019;38:39.

14. Kim TH, Choi JY, Lee HH, Park Y. Associations between dietary pattern and depression in Korean adolescent girls. J Pediatr Adolesc Gynecol 2015;28(6):533-537.

15. Oh J, Chung J. Fruit and vegetable consumption frequency and mental health in Korean adolescents: based on the 2014-2017 Korea Youth Risk Behavior Survey. J Nutr Health 2020;53:518-531 (Korean).

16. Korea Foundation for Suicide Prevention. Suicide prevention white- book 2020 [cited 2020 Dec 14]. Available from: https://spckoreastat.or.kr/boardpublishlist.do (Korean).

17. Williamson C. Dietary factors and depression in older people. $\mathrm{Br}$ J Community Nurs 2009;14:422-426.

18. Wahlqvist ML, Saviage GS. Interventions aimed at dietary and lifestyle changes to promote healthy aging. Eur J Clin Nutr 2000; 54 Suppl 3:S148-S156.

19. Kim JH, Hong YC. GSTM1, GSTT1, and GSTP1 polymorphisms and associations between air pollutants and markers of insulin resistance in elderly Koreans. Environ Health Perspect 2012;120: 1378-1384.

20. Bae JN, Cho MJ. Development of the Korean version of the Geriatric Depression Scale and its short form among elderly psychiatric patients. J Psychosom Res 2004;57:297-305.

21. Lim Y, Oh SY. Development of a semi-quantitative food frequency questionnaire for pre-school children in Korea. Korean J Community Nutr 2002;7:58-66 (Korean).

22. Kim KN, Lee MR, Choi YH, Hwang H, Oh SY, Park C, et al. Association between phthalate exposure and lower handgrip strength in an elderly population: a repeated-measures study. Environ Health 2016;15:93.

23. Korean Society for the Study of Obesity. Common sense for obesity [cited 2021 Jan 26]. Available from: http://general.kosso.or.kr/ html/?pmode = obesity-Diagnosis (Korean).

24. World Health Organization. The Asia-Pacific perspective: redefining obesity and its treatment; 2000 [cited 2021 Jan 26]. Available from: https://apps.who.int/iris/handle/10665/206936.

25. Lee KW, Cho MS. The traditional Korean dietary pattern is associated with decreased risk of metabolic syndrome: findings from the Korean National Health and Nutrition Examination Survey, 1998-2009. J Med Food 2014;17:43-56.

26. Blanchflower DG, Oswald AJ, Stewart-Brown S. Is psychological well-being linked to the consumption of fruit and vegetables? Soc Indic Res 2013;114:785-801.

27. Maes M, De Vos N, Pioli R, Demedts P, Wauters A, Neels H, et al. Lower serum vitamin $\mathrm{E}$ concentrations in major depression. Another marker of lowered antioxidant defenses in that illness. J Affect Disord 2000;58:241-246.

28. Joshi YB, Praticò D. Lipid peroxidation in psychiatric illness: overview of clinical evidence. Oxid Med Cell Longev 2014;2014: 828702.

29. Miller AL. The methylation, neurotransmitter, and antioxidant connections between folate and depression. Altern Med Rev 2008; 13:216-226.

30. Kim JM, Stewart R, Kim SW, Yang SJ, Shin IS, Yoon JS. Predictive value of folate, vitamin B12 and homocysteine levels in late-life depression. Br J Psychiatry 2008;192:268-274.

31. Williams AL, Cotter A, Sabina A, Girard C, Goodman J, Katz DL. The role for vitamin B-6 as treatment for depression: a systematic review. Fam Pract 2005;22:532-537.

32. Westover AN, Marangell LB. A cross-national relationship between sugar consumption and major depression? Depress Anxiety 2002; 16:118-120. 